\title{
PHOTOVOLTAICS
}

\section{Perovskites tower up}

ACS Energy Lett. 3, 2052-2058 (2018)

Perovskite solar cells are receiving great attention recently thanks to their rapid increase in performance as single-junction cells and, even more interestingly, as doublejunction devices. As also happened for III-V semiconductor-based solar cells, more complex multi-junction architectures are being forecast for perovskites and recently a first theoretical exploration of allperovskite and perovskite-silicon triplejunction designs based on optical and electrical simulations was reported. Now, Jerémie Werner and colleagues at the EPFL and CSEM in Switzerland demonstrate experimentally a proof-of-concept twoterminal perovskite-perovskite-silicon triple-junction solar cell, where the mixed cations/mixed halides perovskites, $\mathrm{CsFAPb}(\mathrm{Br}, \mathrm{I})_{3}$, are deposited on a textured silicon bottom cell via sequential deposition.

By varying the content of $\mathrm{Cs}$ and $\mathrm{Br}$, the researchers tune the bandgap to $1.8 \mathrm{eV}$ for the perovskite top cell and to $1.53 \mathrm{eV}$ for the perovskite middle cell. Indium zinc oxide and nanocrystalline hydrogenated silicon are used as recombination junctions between the top/middle and middle/bottom sub-cells, respectively. The triple-junction cell delivers an open-circuit voltage of $2.7 \mathrm{~V}$ and a total current density of $38.8 \mathrm{~mA} \mathrm{~cm}^{-2}$, which translate into an overall efficiency of $13.2 \%$. As the researchers point out, the photovoltaic performance is still far from being optimal and further improvements are needed on the recombination layers, as well as on the layer thicknesses and bandgap tuning of the perovskite absorbers. Still, these findings are a step forward in perovskite-based photovoltaics as they potentially enable efficiencies beyond the thermodynamic limit of single-junction devices.

Giulia Tregnago

Published online: 10 September 2018 https://doi.org/10.1038/s41560-018-0250-9 